\title{
Crosstalk Between Dietary Pattern, Anthropometric Parameters, and Adiponectin Concentration Among Patients with Metabolic Syndrome
}

\author{
Ewelina Pałkowska-Goździk, PhD, Katarzyna Piotrowicz, MD, PhD, Paweł Krzesiński, MD, PhD, \\ Adam Stańczyk, MD, PhD, Andrzej Skrobowski, MD, PhD, and Grzegorz Gielerak, MD, PhD
}

\begin{abstract}
Background: Adiponectin is known to mediate antidiabetic and cardioprotective metabolic effects. Current evidence suggests that diet, both its quantity and quality, affects adiponectin concentration.

Methods: The purpose of this study was to evaluate the association of adiponectin with cardiometabolic risk factors, anthropometric features, and diet characteristics in obese patients with multiple cardiometabolic risk factors. The group of 113 nondiabetic patients (90 males, mean age: $48 \pm 9$ years) fulfilling the criteria of metabolic syndrome (IDF, 2005) was divided into 2 subgroups according to median adiponectin concentration calculated for that cohort $(6.83 \mu \mathrm{g} / \mathrm{mL})$ - low-adiponectin (LA) and high-adiponectin (HA) subgroup. Biochemical and anthropometric measurements were recorded and nutrients intake was assessed using 24-hr dietary recall method and diet history questionnaire.

Results: No significant differences between LA and HA were noted regarding the anthropometric parameters. However, there were significant differences in biochemical indices, and HA was characterized by a more favorable insulin level, homeostasis model assessment of insulin resistance (HOMA-IR) value, and plasma lipid profile than LA. Both LA and HA subgroups were comparable in terms of energy, protein, carbohydrate, and total fat intake, but there were significant differences between the level of polyunsaturated fatty acids (PUFA) consumption (6.06 \pm 1.0 and $6.37 \pm 1.1$, respectively) and omega-3/omega- 6 ratio (as follows: $0.09 \pm 0.01$ and $0.16 \pm 0.04)$.

Conclusions: These results highlight interactions between diet, adiponectin concentration, and biochemical profile. Nondiabetic patients with metabolic syndrome with higher PUFA consumption, including higher omega-3/omega-6 ratio, were characterized by higher circulating adiponectin level and more favorable biochemical profile. Thus, it might be assumed that the protective role of omega- 3 fatty acids might be mediated by adiponectin in patients with metabolic syndrome.
\end{abstract}

Keywords: adiponectin, diet, anthropometric parameters, obesity, metabolic syndrome

\section{Introduction}

L ONG-TERM IMBALANCED high-energy diet combined with low physical activity level consequently lead to pathological accumulation of adipose tissue, which in turn increases the risk of chronic diseases and markedly reduces life expectancy. ${ }^{1}$ Abdominal obesity is a key diagnostic criterion of metabolic syndrome linking other reversible cardiometabolic risk factors such as hypertension, atherogenic dyslipidemia, and glucose intolerance followed by low-grade inflammatory condition ${ }^{2}$ and a prothrombotic state. ${ }^{3}$ Metabolic syndrome is an emerging clinical challenge closely associated with the development of diabetes type $2,{ }^{4}$ cardiovascular diseases, and increased risk of cardiac events ${ }^{5}$ that requires a multifactorial and multidisciplinary approach.

Among adipose tissue-derived hormones, adiponectin plays a special role in the pathophysiology of metabolic syndrome. ${ }^{6,7}$ Accumulating pieces of evidence suggest that adiponectin expresses antioxidant, anti-inflammatory, and antiatherogenic properties that improve insulin sensitivity and endothelial function. ${ }^{8}$ Its concentration is negatively correlated with body visceral fat content and thus presents in

Cardiology and Internal Medicine Department, Military Institute of Medicine, Warsaw, Poland. 
lower concentration in obese individuals. ${ }^{9}$ Hypoadiponectinemia is assumed to be a predictor of obesity-related disorders such as insulin resistance and diabetes, ${ }^{10}$ hypertension, ${ }^{11}$ dyslipidemia, ${ }^{12}$ and left ventricular hypertrophy. ${ }^{13}$ On the contrary, high-adiponectin (HA) concentration provides protection against metabolic syndrome and its complications. ${ }^{14}$

Current evidence suggests that diet, both its quantity and quality, affects adiponectin concentration. ${ }^{15,16}$ It was shown that dietary pattern characterized by high intake of grain and low-fat dairy products was positively associated with adiponectin concentration. ${ }^{17}$ Adherence to the Mediterranean diet, ${ }^{18}$ diet rich in $n-3$ polyunsaturated fatty acids (PUFA), ${ }^{19}$ and significant weight loss (obtained with a low-caloric diet alone or followed by an exercise plan) were also effective in increasing hormone levels. ${ }^{20,21}$ Therefore, the aim of our secondary data analysis study was to evaluate the association of adiponectin with cardiometabolic risk factors, anthropometric features, and diet characteristics in nondiabetic obese patients with multiple cardiometabolic risk factors. Findings from this study might be helpful to design better-targeted strategy to both prevent and treat obesityrelated disorders in the moderate-to-high risk group for cardiovascular events.

\section{Methods}

\section{Subjects}

Among a total of 900 patients consulted in out-patient clinic between December 2010 and December 2013, a group of 130 consecutive patients with ambulatory diagnosed metabolic syndrome (IDF, 2005) ${ }^{22}$ according to detailed inclusion/exclusion criteria were recruited. The patients had no diabetes, history of chronic kidney disease (GFR $>60 \mathrm{~mL} / \mathrm{min} / 1.73 \mathrm{~m}^{2}$ ), thyroid dysfunction, or cardiovascular disease (except arterial hypertension) defined by documented cardiovascular disease (CVD), which includes previous acute myocardial infarction (AMI), acute coronary syndrome (ACS), coronary revascularization, stroke and transient ischemic attack (TIA), aortic aneurysm, and peripheral artery disease (PAD). We excluded patients with diabetes based on European Guidelines on Prevention (2016) where patients with diabetes mellitus are already in high risk or very-high risk group of CVD, which automatically draws the attention of clinicians and the patients themselves. In contrast, patients without diabetes, although presenting risk factors as in the metabolic syndrome are not clinically monitored and they underestimate their health risks.

Finally, after obtaining written informed consent, a total of 113 patients (90 males, 23 females: aged $48 \pm 9$ years) were admitted to the Department of Cardiology and Internal Medicine for the further evaluation.

The study was performed both in accordance with the Declaration of Helsinki and with the approval of the local Ethics Committee (No. 44/WIM/2010). Medical history was taken from all participants, followed by physical examination and anthropometric measurements, including body composition, nutritional assessment, and complete blood profile. Female subjects were postmenopausal (unintended feature) and were not taking hormone replacement therapy.

\section{Biochemical measurements}

Blood samples were collected after an overnight fast by venipuncture into glass tubes without anticoagulant. All biochemical measurements were performed in a core laboratory in the Military Institute of Medicine (Warsaw, Poland). Samples were centrifuged at $2000 \mathrm{~g}$ for $15 \mathrm{~min}$ at the temperature of $40^{\circ} \mathrm{C}$ to obtain serum. Fasting glucose, total cholesterol (TC), high- and low-density lipoproteins cholesterol (HDL-C and LDL-C, respectively), and triglycerides (TG) were determined in serum by standard methods with the use of Roche reagents and Integra 800 analyzer. Apolipoprotein B (ApoB) and apolipoprotein A1 (ApoA1) were determined by the immunoturbidimetric method with the use of the same analyzer. High-sensitivity C-reactive protein (hsCRP) was determined with a highly sensitive immunonephelometric method using Siemens reagents (CardioPhase ${ }^{\circledR}$ hsCRP) and BN2 analyzer. Insulin was determined by the electrochemiluminescence immunoassay with Roche Cobas 6000 analyzer. Coefficients of variation $(\mathrm{CVs})$ for the above analytes were from $5 \%$ to $9 \%$.

Insulin resistance was quantified according to HOMA (homeostatic model assessment) proposed by Mathius using following equation: HOMA-IR $=[$ insulin $(\mu \mathrm{U} / \mathrm{mL}) \times$ glucose $(\mathrm{mg} / \mathrm{dL})] / 405$.

Adipocyte proteins were determined by enzyme-linked immunosorbent assay (ELISA). Serum adiponectin was determined with ready to use Human Adiponectin Platinum ELISA Reagent Kits from eBioscience (CV 4.2\%). Leptin was determined in serum using Leptin (Sandwich) ELISA Reagent Kits from DRG International, Inc. (CV 5.1\%).

\section{Anthropometrics and body composition}

Detailed information concerning measurement of weight, height, body composition, and waist circumference (WC) has been previously reported. ${ }^{18}$ In brief, whole-body mass, fat mass (FM), fat-free mass (FFM), and basal metabolic rate were obtained using Body Composition Analyzer BC418 (Tanita, The Netherlands). All measurements were taken in morning hours (before breakfast). Participants were without shoes and wore light clothes. WC was measured at the narrowest circumference between the bottom of the rib cage and the iliac crest (at the level of the umbilicus). Hip circumference was measured at the widest girth of the buttocks. Both measurements were taken by using the same unstretching tape measure for each patient and were recorded to the nearest $0.1 \mathrm{~cm}$. In the current study, general obesity was defined as WC $\geq 80$ and $\geq 94 \mathrm{~cm}$ for women and men, respectively. ${ }^{22}$

\section{Nutritional assessment}

As it was previously reported, ${ }^{23}$ patients' food intake and eating habits were evaluated using the 24-hr dietary recall method and an author's semiquantitative diet history questionnaire, in which questions were concerning the amount and the frequency of each products groups consumption (fruit and vegetables, whole grain products, eggs, milk and milk products, meat, fish, starch and nuts). The declaration of dietary supplements use and cooking techniques were also recorded. Respondents were asked to indicate an average size of commonly consumed food portion. Standard serving sizes were provided as a basic information for further estimations (photo album of products, and dishes were shown). Participants were interviewed during individual consultations provided exclusively by the same dietitian. 
Information about the nutritional composition of the consumed diet was based on Dieta 4.0 software (Institute of Food and Nutrition, Poland). The average daily energy, fat, saturated fatty acids (SFA) and PUFA, carbohydrate, sucrose, protein, and dietary fiber intake was assessed. The dietary polyunsaturated omega- 3 to omega- 6 fatty acids ratio was also calculated. Estimated total energy intake (TEI) was presented as kilocalorie per kilogram of fat-free mass [kcal/kg FFM], nutrients intake as percentage of total energy intake [\% TEI], and dietary fiber-grams per 100 kcal diet energy.

\section{Statistical analysis}

All results are expressed as the mean \pm standard deviation or number $(\%)$ in case of categorical data. Statistical analysis was performed using STATISTICA 12.0 (StatSoft). The study population was divided into two groups-low adiponectin (LA) and HA (median adiponectin concentration amounting to $6.83 \mu \mathrm{g} / \mathrm{mL}$ in studied population). Comparison of the groups was examined by one-way ANOVA. NIR Fisher test was used for post hoc analysis and correlations between variables were assessed by Pearson test and $r$ coefficients were presented. Significance was set at $P<0.05$.

\section{Results}

\section{Basic characteristics}

Metabolic and anthropometric characteristics of 90 men and 23 women are summarized in Table 1. Data concerning concentrations of total, LDL-C, HDL-C, TG, and \% FM values were previously described. ${ }^{23}$

\section{Correlations between adiponectin, metabolic indices, and dietary intake}

Inverse correlations between adiponectin concentration and both TC/HDL $(r=-0.28, P<0.05)$ and TG/HDL ratios $(r=-0.29, P<0.05)$ were present. A strong positive relationship between adiponectin and the ApoA1 level was noted $(r=0.55, P<0.001)$. Furthermore, adiponectin was positively correlated with fat mass index (FMI) $(r=0.30$, $P<0.01)$ and negatively with FFM index $(r=0.25, P<0.05)$. Data regarding selected dietary intake of participants are presented in Table 2. Detailed analysis showed a significant negative correlation between serum adiponectin levels and SFA intake $(r=-0.30, P<0.05)$. However, hormone concentration was positively related to PUFA consumption $(r=0.29, P<0.05)$ and omega-3/omega-6 FA ratio $(r=0.27$, $P<0.05)$.

\section{Comparison between LA and HA group}

Clinical, biochemical, and anthropometric indices of both subgroups are shown in Table 3 . No significant differences between LA and HA were noted regarding the anthropometric parameters. However, there were significant differences in biochemical indices, and HA was characterized by a more favorable insulin level, homeostasis model assessment of insulin resistance (HOMA-IR) value, and plasma lipid profile than LA (Table 3).

Although both subgroups were comparable in terms of energy, protein, carbohydrate, and total fat intake, there was
Table 1. Metabolic And Anthropometric PARAmeters of the Study Population

\begin{tabular}{lc}
\hline Monitored parameters & Mean $\pm S D$ \\
\hline Metabolic indices & \\
Adiponectin, $\mu \mathrm{g} / \mathrm{L}$ & $7.85 \pm 5.5$ \\
Insulin, mU/L & $15.70 \pm 7.5$ \\
HOMA-IR & $3.88 \pm 2.1$ \\
Leptin, ng/mL & $15.13 \pm 7.3$ \\
Leptin/adiponectin ratio & $2.67 \pm 2.4$ \\
TC/HDL-C ratio & $5.52 \pm 1.4$ \\
LDL/HDL-C ratio & $3.27 \pm 1.0$ \\
TG/HDL-C ratio & $6.10 \pm 3.7$ \\
ApoA1, g/L & $1.56 \pm 0.3$ \\
ApoB, g/L & $1.11 \pm 0.3$ \\
ApoB/A1 ratio & $0.72 \pm 0.2$ \\
hsCRP, mg/L & $0.24 \pm 0.2$ \\
Systolic BP, mmHg & $141 \pm 17$ \\
Diastolic BP, mmHg & $90 \pm 10$ \\
BMR, kcal/day & $2050 \pm 372.7$ \\
Anthropometric indices & \\
Body weight, kg & $100.4 \pm 14.8$ \\
BMI, kg/m ${ }^{2}$ & $32.7 \pm 4.0$ \\
WC, cm & $110.5 \pm 9.0$ \\
HC, cm & $103.4 \pm 8.9$ \\
WHtR & $0.63 \pm 0.1$ \\
WHI & $1.09 \pm 0.1$ \\
FMI, kg/m ${ }^{2}$ & $9.45 \pm 2.9$ \\
FFMI, kg/m ${ }^{2}$ & $23.10 \pm 2.6$ \\
\hline
\end{tabular}

Apo, apolipoprotein; BMI, body mass index; BMR, basal metabolic rate; BP, blood pressure; FFMI, fat-free mass index (fat-free mass/height squared); FMI, fat mass index (fat mass/height squared); HC, hip circumference; HDL-C, high-density lipoprotein cholesterol; HOMA-IR, homeostasis model assessment of insulin resistance; hsCRP, high-sensitivity C-reactive protein; LDL-C, lowdensity lipoprotein cholesterol; SD, standard deviation; TC, total cholesterol; TG, triglycerides; WC, waist circumference; WHI, waist-to-hip ratio; WHtR, waist circumference-to-height ratio.

a discrepancy between the level of PUFA consumption and omega-3/omega-6 FA ratio (Table 4).

\section{Discussion}

Our analysis revealed that adiponectin concentration is related to dietary habits, anthropometric parameters, and biochemical disturbances. The correlation with FM, expressed as a FMI, confirmed its adipose tissue origin. The primary finding is that patients with a higher concentration of adiponectin had a better cardiometabolic profile and their

Table 2. Nutritional Assessment

\begin{tabular}{lc}
\hline Monitored parameters & Mean $\pm S D$ \\
\hline Energy, kcal/kg FFM & $37.3 \pm 8.3$ \\
Protein, \% TEI & $14.6 \pm 1.7$ \\
Fat, \% TEI & $36.2 \pm 5.7$ \\
SFA, \% TEI & $12.6 \pm 2.0$ \\
PUFA, \% TEI & $6.2 \pm 1.7$ \\
Omega-3/omega-6 FA ratio & $0.12 \pm 0.05$ \\
Carbohydrate, \% TEI & $48.5 \pm 6.3$ \\
Sucrose, \% TEI & $10.2 \pm 1.5$ \\
Dietary fiber, g/100 kcal diet energy & $0.8 \pm 0.14$ \\
\hline
\end{tabular}

FA, fatty acids; FFM, fat-free mass; PUFA, polyunsaturated fatty acids; SFA, saturated fatty acids; TEI, total energy intake. 
TABle 3. COMParison OF Clinical, Laboratory, AND ANTHRopometry Parameters Between LOW- AND High-AdIPONECTIN GROUPS

\begin{tabular}{|c|c|c|c|}
\hline $\begin{array}{l}\text { Monitored } \\
\text { parameters }\end{array}$ & $\begin{array}{l}L A(\mathrm{n}=61) \\
\text { mean } \pm S D\end{array}$ & $\begin{array}{c}H A(\mathrm{n}=52) \\
\text { mean } \pm S D\end{array}$ & $\mathrm{P}$ \\
\hline \multicolumn{4}{|l|}{ Clinical indices } \\
\hline Females/males & $11 / 50$ & $12 / 40$ & - \\
\hline Age, years & $47 \pm 7$ & 49 & NS \\
\hline \multicolumn{4}{|l|}{ Metabolic indices } \\
\hline $\begin{array}{l}\text { Adiponectin, } \\
\mu \mathrm{g} / \mathrm{L}\end{array}$ & $4.20 \pm 1.5$ & $11.60 \pm 5.5$ & $<0.001$ \\
\hline Insulin, mU/l & $16.41 \pm 4.7$ & $13.15 \pm 3.8$ & $<0.05$ \\
\hline HOMA-IR & $4.3 \pm$ & 3.18 & $<0.05$ \\
\hline Leptin, ng/mL & $14.33 \pm 6.1$ & $15.95 \pm 7.1$ & NS \\
\hline $\begin{array}{l}\text { Leptin/ } \\
\text { adiponectin } \\
\text { ratio }\end{array}$ & $3.75 \pm 2.7$ & $1.56 \pm 1.5$ & $<0.001$ \\
\hline TC/HDL ratio & $5.66 \pm 1.4$ & $5.37 \pm 1.4$ & NS \\
\hline LDL/HDL ratio & $3.43 \pm 1.0$ & $2.76 \pm 1.0$ & $<0.05$ \\
\hline TG/HDL ratio & $6.33 \pm 3.6$ & $5.80 \pm 3.8$ & $<0.05$ \\
\hline ApoA1, g/L & $1.43 \pm 0.2$ & $1.68 \pm 0.2$ & $<0.001$ \\
\hline ApoB, g/L & $1.11 \pm 0.3$ & $1.12 \pm 0.3$ & NS \\
\hline ApoB/A1 & $0.78 \pm 0.2$ & $0.67 \pm 0.2$ & $<0.05$ \\
\hline hsCRP, mg/L & $0.24 \pm 0.1$ & $0.24 \pm 0.1$ & NS \\
\hline BMR, kcal/day & $2165.2 \pm 340.8$ & $1988.1 \pm 373.4$ & NS \\
\hline \multicolumn{4}{|c|}{ Anthropometric indices } \\
\hline Body weight, $\mathrm{kg}$ & $101.8 \pm 15.1$ & $98.9 \pm 14.2$ & NS \\
\hline BMI, $\mathrm{kg} / \mathrm{m}^{2}$ & $32.3 \pm 4.2$ & $33.2 \pm 3.6$ & NS \\
\hline $\mathrm{WC}, \mathrm{cm}$ & $109.1 \pm 10.5$ & $112.4 \pm 8.8$ & NS \\
\hline $\mathrm{HC}, \mathrm{cm}$ & $102.3 \pm 8.9$ & $104.6 \pm 8.3$ & NS \\
\hline WHtR & $0.62 \pm 0.1$ & $0.64 \pm 0.05$ & NS \\
\hline WHI & $1.08 \pm 0.1$ & $1.09 \pm 0.1$ & NS \\
\hline FMI, $\mathrm{kg} / \mathrm{m}^{2}$ & $8.92 \pm 2.9$ & $10.08 \pm 2.7$ & NS \\
\hline FFMI, $\mathrm{kg} / \mathrm{m}^{2}$ & $23.46 \pm 2.0$ & $22.55 \pm 3.2$ & NS \\
\hline
\end{tabular}

LA/HA, low- and high-adiponectin group, respectively; NS, not significant.

diet characterized with higher PUFA consumption followed by more favorable dietary fatty acids ratio than those from LA group despite the lack of differences in the values of anthropometric indices between the groups. Therefore, the question arises whether adiponectin mediates beneficial effects of PUFA omega-3 fatty acids or expresses dietary-

\section{Table 4. Comparison of Dietary Assessment PARAMETERS BETWEen Low- AND High-ADIPONECTIN Groups}

\begin{tabular}{lccc}
\hline $\begin{array}{l}\text { Monitored } \\
\text { parameters }\end{array}$ & $\begin{array}{c}\text { LA }(\mathrm{n}=61), \\
\text { mean } \pm S D\end{array}$ & $\begin{array}{c}H A \\
(\mathrm{n}=52), \\
\text { mean } \pm S D\end{array}$ & $\mathrm{P}$ \\
\hline Energy, kcal/kg FFM & $36.39 \pm 5.7$ & $38.36 \pm 9.6$ & $\mathrm{NS}$ \\
Protein, \% TEI & $14.48 \pm 2.0$ & $14.70 \pm 1.3$ & $\mathrm{NS}$ \\
Fat, \% TEI & $36.07 \pm 3.6$ & $36.35 \pm 7.5$ & $\mathrm{NS}$ \\
SFA, \% TEI & $13.45 \pm 2.0$ & $12.67 \pm 2.0$ & $\mathrm{NS}$ \\
PUFA, \% TEI & $6.06 \pm 1.0$ & $6.37 \pm 1.1$ & $<0.05$ \\
Omega-3/omega-6 FA & $0.09 \pm 0.01$ & $0.16 \pm 0.04$ & $<0.05$ \\
$\quad$ ratio & & & \\
$\begin{array}{l}\text { Carbohydrates, \% TEI } \\
\text { Sucrose, \% TEI }\end{array}$ & $49.20 \pm 4.6$ & $47.75 \pm 7.8$ & $\mathrm{NS}$ \\
Dietary fiber, g/100 kcal & $9.61 \pm 2.0$ & $10.52 \pm 0.5$ & $\mathrm{NS}$ \\
$\quad$ & $0.77 \pm 0.15$ & $0.88 \pm 0.13$ & $\mathrm{NS}$ \\
diet energy & & & \\
\hline
\end{tabular}

independent positive action on metabolic abnormalities. Due to the potential multidirectional beneficial effects of adiponectin on cardiovascular health, recognition of modifiable predictors of hormone concentrations would be of major interest.

It is well documented that hypoadiponectinemia is closely associated with the clinical phenotype of metabolic syndrome $^{24}$ and our results corroborate those findings. Lower adiponectin levels in obese patients are associated with significantly higher insulin concentration, HOMA value, TC/HDL, LDL/HDL, and TG/HDL ratios compared with the HA group. Variations in adiponectin levels affect individual risk stratification of cardiovascular diseases and diabetes type 2 . The reduction in plasma adiponectin level has been shown to contribute to insulin resistance development. ${ }^{25}$ Thus, the adiponectin insulin-sensitizing effect could be explained, at least in part, by stimulation of AMPactivated protein kinase (AMPK) in both skeletal muscle and the liver. ${ }^{26}$ Furthermore, the molecular mechanism by which adiponectin may influence insulin action involves increased muscle fat oxidation, glucose uptake via AMPKactivation, and acetyl-CoA carboxylase inhibition. ${ }^{27}$ Adiponectin limits endogenous glucose production in the liver by inhibiting the expression of gluconeogenic enzymes such as phosphoenolpyruvate carboxykinase and glucose-6phosphatase. $^{28,29}$

Both the atherogenic index (TC/HDL-C ratio), the LDL/HDL and TG/HDL-C ratios, are recognized as meaningful indicators of insulin resistance and cardiovascular risk and their predictive values are supposed to be greater than single parameters. ${ }^{12,30,31}$ According to risk categories for TC/HDL-C and LDL-C/HDL-C ratios, in primary prevention, target levels are estimated to be lower than 4.5 in men and 4 in women and lower than 3.0 in men and 2.5 in women, correspondingly. ${ }^{31}$ Although those two parameters exceed the specified limits in the study population, inverse correlations between adiponectin concentration and both TC/HDL and TG/HDL ratios were found. Our observations are in line with previous studies indicating the relationship between adiponectin and plasma lipid economy, especially HDL-C concentration. ${ }^{12}$ It was found that hormone concentration was positively correlated with HDL-C level independently of body mass index ${ }^{12}$ which probably resulted from stimulating effect of adiponectin on the activity of peroxisome proliferation-activated receptor $\alpha(\operatorname{PPAR} \alpha)$ in both liver and skeletal muscle. PPAR $\alpha$ upregulates the expression of apoproteins $\mathrm{A} 1$ and $\mathrm{A} 2$ in the liver, which in turn increases hepatic HDL-C production and secretion. ${ }^{32}$ Results of the presented study are concordant with a study that has shown a strong positive correlation between plasma adiponectin level and ApoA1 concentration. ${ }^{33}$ Moreover, the HA group was also characterized by higher ApoB/A1 ratio than LA. Our findings are compatible with current hypothesis stressing the role of ApoB/A1 ratio as a strong predictor of atherosclerotic vascular diseases. ${ }^{31,34}$ Furthermore, higher ApoB/A1 ratio is significantly associated with insulin resistance. ${ }^{35}$ Therefore, it can be assumed that HA level in HA group may limit cardiometabolic risk variables for type 2 diabetes and cardiovascular disease.

Production and secretion of adiponectin are regulated by two, endogenous and exogenous, factors. The first group includes oxidative stress in adipose tissue, insulin, testosterone, prolactin, growth hormone, glucocorticoids, and cytokines- 
divided adipose tissue (IL-6, IL-8, and TNF- $\alpha$ ) ${ }^{36,37}$ Lifestyle elements such as smoking status, physical activity levels, diet behaviors (energy supply and diet composition), and a number of medications (including angiotensin receptor type 1 blockers, angiotensin-converting enzyme (ACE) inhibitors, and cannabinoid receptor antagonists) are environmental factors that act as modulators of adiponectin concentration.

Evidence suggests that caloric restriction and weight reduction are able to increase plasma adiponectin level in obese diabetic patients. ${ }^{38}$ Adherence to a Mediterranean diet - considered as a gold standard for heart disease prevention-is associated with high concentration of adiponectin in healthy ${ }^{18}$ and diabetic individuals. ${ }^{39}$ Several components of the diet are worth attention due to their positive correlation with adiponectin level, primarily fruit, nuts, whole-grain products, and moderate alcohol consumption. ${ }^{39}$ The mechanisms of Mediterranean diet stimulating effects are not fully understood, probably it may project its antioxidant and anti-inflammatory properties. ${ }^{40}$

In our study, diets of LA and HA groups were characterized by higher SFA intake and lesser fiber content than recommended. ${ }^{41}$ Although the percentage of main macronutrients (carbohydrates, proteins, and fats) in both diets was similar, the level of PUFA intake was the nutritional factor differentiating the groups. Those observations support the notion that diet rich in omega-3 or supplementation with omega-3 is effective in increasing adiponectin concentration both in human studies and using animals models. ${ }^{42-45} \mathrm{We}$ found that not only PUFA quantity but also quality affects adiponectin level. HA group was characterized by higher omega-3/omega-6 ratio. A similar conclusion was made by Guebre-Egziabher et al. ${ }^{46}$ After 10 -week nutritional intervention designed to the simultaneous increase of omega-3 fatty acids and decrease omega- 6 fatty acid intake, a reduction in LDL-C, plasma TNF- $\alpha$, and increase in lipid oxidation and plasma adiponectin concentration was found. ${ }^{46}$ The higher adiponectin levels promoted (at least partially) by higher omega- 3 intake appears to mediate the modulation in insulin sensitivity as HA group was characterized by lower insulin level, HOMA-IR value and a more favorable plasma lipid profile than LA.

Mechanisms that may underlie our results include the n-3 PUFA role in regulation of key enzymes activity involved in fatty acid oxidation, ${ }^{47}$ and modulation of adiponectin expression through AMPK stimulation in the liver and adipocytes $^{44}$ or interaction with PPAR $^{48}$ to reduce obesityinduced insulin resistance. ${ }^{49,50}$

Due to the importance of adiponectin in the prevention of cardiometabolic disturbances, the identification, and implementation of interventions that prevent hypoadiponectinemia should become a desired target for future therapeutic strategy. In a view of our results ensuring proper supply of omega-3 fatty acids followed by cardioprotective diet plan seems to be an effective approach that might limit the degree of metabolic syndrome components in clinical practice.

\section{Limitations}

The limited number of subjects and the unbalance between sexes (especially lack of premenopausal women) should be noted. There is evidence indicating adiponectin concentration to be gender-dependent-higher among women than men. This is believed to be attributed to the in- hibitory effect of the testosterone on adiponectin secretion from adipocytes. Our results were obtained in a particular group of patients (with nondiabetic metabolic syndrome) and should be carefully extrapolated to the general population. It should also be taken into account that the hormonal status of women was not controlled in this study.

\section{Conclusions}

Adiponectin concentration is related (at least partially) to dietary habits, and atherogenic biochemical profile. Nondiabetic patients with metabolic syndrome with a higher concentration of adiponectin had a better cardiometabolic profile and their diet characterized with higher PUFA consumption followed by more favorable dietary fatty acids ratio. Thus, our observations confirm that nutritional factors are important aspects of therapeutic strategy for managing cardiometabolic disorders in patients with metabolic syndrome. The influence of diet on adiponectin concentration and its beneficial metabolic effects should be further investigated.

\section{Acknowledgments}

The authors thank the participants of this study, the research staff, and reviewers for comments.

\section{Author Disclosure Statement}

No conflicting financial interests exist.

\section{Funding Information}

The study was supported by MNiSW/WIM statutory grant number 69.

\section{References}

1. Grover SA, Kaouache M, Rempel P, et al. Years of life lost and healthy life-years lost from diabetes and cardiovascular disease in overweight and obese people: A modeling study. Lancet Diabetes Endocrinol 2015;3:114-122.

2. Monteiro R, Azevedo I. Chronic inflammation in obesity and the metabolic syndrome. Mediators Inflamm 2010; 2010:289645.

3. Kaur J. A comprehensive review on metabolic syndrome. Cardiol Res Pract 2014;2014:943162.

4. Shin JA, Lee JH, Lim SY, et al. Metabolic syndrome as a predictor of type 2 diabetes, and its clinical interpretations and usefulness. J Diabetes Investig 2013;4:334-343.

5. Kazlauskiené L, Butnorienè J, Norkus A. Metabolic syndrome related to cardiovascular events in a 10-year prospective study. Diabetol Metab Syndr 2015;7:102.

6. Ebrahimi-Mamaeghani M, Mohammadi S, Arefhosseini $\mathrm{SR}$, et al. Adiponectin as a potential biomarker of vascular disease. Vasc Health Risk Manag 2015;11:55-70.

7. Tajtákova M, Petrásova D, Petrovicová J, et al. Adiponectin as a biomarker of clinical manifestation of metabolic syndrome. Endocr Regul 2006;40:15-19.

8. Achari AE, Jain SK. Adiponectin, a therapeutic target for obesity, diabetes, and endothelial dysfunction. Int J Mol Sci 2017;18:1312.

9. Kawano J, Arora R. The role of adiponectin in obesity, diabetes, and cardiovascular disease. J Cardiometab Syndr 2009;4:44-49. 
10. Xita N, Tsatsoulis A. Adiponectin in diabetes mellitus. Curr Med Chem 2012;19:5451-5458.

11. Rojas E, Rodríguez-Molina D, Bolli $\mathrm{P}$, et al. The role of adiponectin in endothelial dysfunction and hypertension. Curr Hypertens Rep 2014;16:463.

12. Izadi V, Farabad E, Azadbakht L. Epidemiologic evidence on serum adiponectin level and lipid profile. Int J Prev Med 2013;4:133-140.

13. Lee Y, Kim BK, Lim YH, et al. The relationship between adiponectin and left ventricular mass index varies with the risk of left ventricular hypertrophy. PLoS One 2013;8: e70246.

14. Kim JY, Yadav D, Ahn SV, et al. A prospective study of serum adiponectin and regression of metabolic syndrome: The ARIRANG study. Biochem Biophys Res Commun 2015;466:201-205.

15. Reis CE, Bressan J, Alfenas RC. Effect of the diet components on adiponectin levels. Nutr Hosp 2010;25:881-888.

16. Lopez-Jaramillo P. The role of adiponectin in cardiometabolic diseases: Effects of nutritional interventions. $J$ Nutr 2016;146:422-426.

17. Yannakoulia M, Yiannakouris N, Melistas L, et al. A dietary pattern characterized by high consumption of wholegrain cereals and low-fat dairy products and low consumption of refined cereals is positively associated with plasma adiponectin levels in healthy women. Metabolism 2008;57:824-830.

18. Fragopoulou E, Panagiotakos DB, Pitsavos C, et al. The association between adherence to the Mediterranean diet and adiponectin levels among healthy adults: The ATTICA study. J Nutr Biochem 2010;21:285-289.

19. Krebs JD, Browning LM, McLean NK, et al. Additive benefits of long-chain n-3 polyunsaturated fatty acids and weight-loss in the management of cardiovascular disease risk in overweight hyperinsulinaemic women. Int J Obes (Lond) 2006;30:1535-1544.

20. Rokling-Andersen MH, Reseland JE, Veierød MB, et al. Effects of long-term exercise and diet intervention on plasma adipokine concentrations. Am J Clin Nutr 2007;86: 1293-12301.

21. Simpson KA, Singh MA. Effects of exercise on adiponectin: A systematic review. Obesity (Silver Spring) 2008; 16:241-256.

22. International Diabetes Federation. The IDF consensus worldwide definition of the metabolic syndrome. Published 2006. Accessed at https://www.idf.org/e-library/consensusstatements/60-idfconsensus-worldwide-definitionof-the-met abolic-syndrome.html on March, 2018.

23. Piotrowicz K, Pałkowska E, Bartnikowska E, et al. Selfreported health-related behaviors and dietary habits in patients with metabolic syndrome. Cardiol J 2015;22:413420.

24. Di Chiara T, Argano C, Corrao S, et al. Hypoadiponectinemia: A link between visceral obesity and metabolic syndrome. J Nutr Metab 2012;2012:175245.

25. Kadowaki T, Yamauchi T, Kubota N, et al. Adiponectin and adiponectin receptors in insulin resistance, diabetes, and the metabolic syndrome. J Clin Invest 2006;116:17841792. Review.

26. Yamauchi T, Kamon J, Minokoshi Y, et al. Adiponectin stimulates glucose utilization and fatty-acid oxidation by activating AMP-activated protein kinase. Nat Med 2002;8: 1288-1295.

27. Tomas E, Tsao TS, Saha AK, et al. Enhanced muscle fat oxidation and glucose transport by ACRP30 globular do- main: Acetyl-CoA carboxylase inhibition and AMPactivated protein kinase activation. Proc Natl Acad Sci U S A 2002;99:16309-16313.

28. Combs TP, Berg AH, Obici S, et al. Endogenous glucose production is inhibited by the adipose-derived protein Acrp30. J Clin Invest 2001;108:1875-1881.

29. Combs TP, Pajvani UB, Berg AH, et al. A transgenic mouse with a deletion in the collagenous domain of adiponectin displays elevated circulating adiponectin and improved insulin sensitivity. Endocrinology 2004;145:367383.

30. Natarajan S, Glick H, Criqui M, et al. Cholesterol measures to identify and treat individuals at risk for coronary heart disease. Am J Prev Med 2003;25:50-57.

31. Millán J, Pintó X, Muñoz A, et al. Lipoprotein ratios: Physiological significance and clinical usefulness in cardiovascular prevention. Vasc Health Risk Manag 2009;5: 757-765.

32. Toth PP. Adiponectin and high-density lipoprotein: A metabolic association through thick and thin. Eur Heart $J$ 2005;26:1579-1581.

33. Kazumi T, Kawaguchi A, Hirano T, et al. Serum adiponectin is associated with high-density lipoprotein cholesterol, triglycerides, and low-density lipoprotein particle size in young healthy men. Metabolism 2004;53:589-593.

34. Park JS, Cho MH, Nam JS, et al. Adiponectin is independently associated with apolipoprotein B to A-1 ratio in Koreans. Metabolism 2010;59:677-682.

35. Matsumoto K, Fujita N, Nakamura K, et al. Apolipoprotein $\mathrm{B}$ and insulin resistance are good markers of carotid atherosclerosis in patients with type 2 diabetes mellitus. Diabetes Res Clin Pract 2008;82:93-97.

36. Swarbrick MM, Havel PJ. Physiological, pharmacological, and nutritional regulation of circulating adiponectin concentrations in humans. Metab Syndr Relat Disord 2008;6: 87-102.

37. Hassanpour SH, Dehghani MA, Alipour SM, et al. Regulation of adiponectin and its receptors by endogenous and exogenous factors. Diabetes Obes Int J 2018;3:000174.

38. Monzillo LU, Hamdy O, Horton ES, et al. Effect of lifestyle modification on adipokine levels in obese subjects with insulin resistance. Obes Res 2003;11:1048-1054.

39. Mantzoros CS, Williams CJ, Manson JE, et al. Adherence to the Mediterranean dietary pattern is positively associated with plasma adiponectin concentrations in diabetic women. Am J Clin Nutr 2006;84:328-335.

40. Richard C, Royer MM, Couture P, et al. Effect of the Mediterranean diet on plasma adipokine concentrations in men with metabolic syndrome. Metabolism 2013;62:18031810.

41. Piepoli MF, Hoes AW, Agewall S, et al. 2016 European Guidelines on cardiovascular disease prevention in clinical practice: The Sixth Joint Task Force of the European Society of Cardiology and Other Societies on Cardiovascular Disease Prevention in Clinical Practice (constituted by representatives of 10 societies and by invited experts) Developed with the special contribution of the European Association for Cardiovascular Prevention \& Rehabilitation (EACPR). Eur Heart J 2016;37:2315-2381.

42. Itoh M, Suganami T, Satoh N, et al. Increased adiponectin secretion by highly purified eicosapentaenoic acid in rodent models of obesity and human obese subjects. Arterioscler Thromb Vasc Biol 2007;27:1918-1925.

43. Kratz M, Swarbrick MM, Callahan HS, et al. Effect of dietary n-3 polyunsaturated fatty acids on plasma total and 
high-molecular-weight adiponectin concentrations in overweight to moderately obese men and women. Am J Clin Nutr 2008;87:347-353.

44. Flachs P, Mohamed-Ali V, Horakova O, et al. Polyunsaturated fatty acids of marine origin induce adiponectin in mice fed a high-fat diet. Diabetologia 2006;49:394-397.

45. Micallef MA, Garg ML. Anti-inflammatory and cardioprotective effects of $n-3$ polyunsaturated fatty acids and plant sterols in hyperlipidemic individuals. Atherosclerosis 2009;204:476-482.

46. Guebre-Egziabher F, Rabasa-Lhoret R, Bonnet F, et al. Nutritional intervention to reduce the $n-6 / n-3$ fatty acid ratio increases adiponectin concentration and fatty acid oxidation in healthy subjects. Eur J Clin Nutr 2008;62: 1287-1293.

47. Baker PW, Gibbons GF. Effect of dietary fish oil on the sensitivity of hepatic lipid metabolism to regulation by insulin. J Lipid Res 2000;41:719-726.

48. Neschen S, Morino K, Rossbacher JC, et al. Fish oil regulates adiponectin secretion by a peroxisome proliferatoractivated receptor-gamma-dependent mechanism in mice. Diabetes 2006;55:924-928.
49. Yamauchi T, Kadowaki T. Physiological and pathophysiological roles of adiponectin and adiponectin receptors in the integrated regulation of metabolic and cardiovascular diseases. Int J Obes (Lond) 2008;32(Suppl 7):13-18.

50. Tsuchida A, Yamauchi T, Takekawa S, et al. Peroxisome proliferator-activated receptor (PPAR)alpha activation increases adiponectin receptors and reduces obesity-related inflammation in adipose tissue: Comparison of activation of PPARalpha, PPARgamma, and their combination. Diabetes 2005;54:3358-3370.

Address correspondence to: Ewelina Patkowska-Goździk, PhD Cardiology and Internal Medicine Department Military Institute of Medicine Szaserow Str. 128 Warsaw 04-141 Poland

E-mail: epalkowska@wim.mil.pl 